\title{
Passion of a Different Kind: Working Career as an Electron Microscopist
}

Mary B. Ard ${ }^{1 *}$

1. Georgia Electron Microscopy/University of Georgia, Athens, GA, USA.

* Corresponding author: maryard@uga.edu

Electron microscopy is used more than ever in the area of veterinary medicine. From negative staining of canine feces to tissue processing and sectioning of sea otter cerebellum, the samples are endless coming from the far side of the Animal Kingdom. There are a number of electron microscopy (EM) facilities across this nation and abroad conducting research involving animal subjects, cell culture and the like with the understanding that the outcome of the research will benefit humanity.

There is another avenue for which some EM facilities "crank out" results, and that is in the area of diagnostics. This presentation is geared toward diagnostic EM in the area of veterinary medicine. A number of my most interesting cases will be discussed - not in their full entirety unless permission is granted by the pathologists, veterinarians, or even owners of these clients who are directly related to these cases. But first, a little bit about basics...

For diagnostic cases, we try to use the same protocol for transmission electron microscopy naming it "routine". Over the years, we have edited this protocol - the editing more in the area of processing based on the tissue and how to enhance the organelles or ultrastructure which will be evaluated. These edits are usually taken from research projects we have done with our clients which gave positive results within their research. One example is using a slightly different protocol for tissue samples submitted for ciliary dyskinesia. This is a condition in young animals, mostly dogs, in which their cilia do not move properly or in sync with one another. At the EM level, the pathologist evaluates the cilia ultrastructure for specific abnormal positioning of the classic $9+2$ arrangement of tubules. In the processing protocol, we add $1 \%$ tannic acid to the glutaraldehyde for one hour before rinsing out the primary fixative and introducing post fixation in osmium tetroxide. We had one of our cilia researchers use this protocol for their research on cilia motility, and their results demonstrating the tubules and dynein arms prompted the decision to use this protocol for our ciliary dyskinesia cases.

Negative staining is a procedure used in electron microscopy which we have been using for this procedure for some of our diagnostic work. Most of the specimens submitted for diagnostic negative staining are fecal material and scabs/crusts. Yes, the samples are not the most ideal to work with, but some have interesting results. This is one of the procedures which I can easily title one of three outcomes: a victory, a standoff, or a defeat. Even the protocols from Standard Operating Procedures and articles suggest a simplified method to view viral particles by electron microscopy. Well, as one who has worked with samples for negative staining a long time in the world of diversified species consistency is key when it pertains to the same type of sample. Consistency meaning the amount of deionized/distilled water added to feces to make the right soupy mixture for proper clarification; or making a lysate from a dried up crusty material which will provide enough information when applied on a grid.

Working in an EM Lab has been rewarding for me. There are few days which are what you call "routine". There is nothing like working a case with a pathologist, or finding a virus in a sample of calf 
feces. It is true for me and my work when we say "it is the little things that matter"! Acknowledgements go out to all the pathologists and residents who submitted cases for diagnostic EM. Without these opportunities, we would not have grown in our knowledge of veterinary pathology [1].

\section{References:}

[1] The author would like to acknowledge all the pathologists who have submitted cases for diagnostic EM from the Pathology Department and the Athens Diagnostic Laboratory here at the University of Georgia. The late Dr. W.L. Steffens is thanked for his years of mentoring this eager microscopist; Dr. John Shields is thanked for his supervisory encouragement.

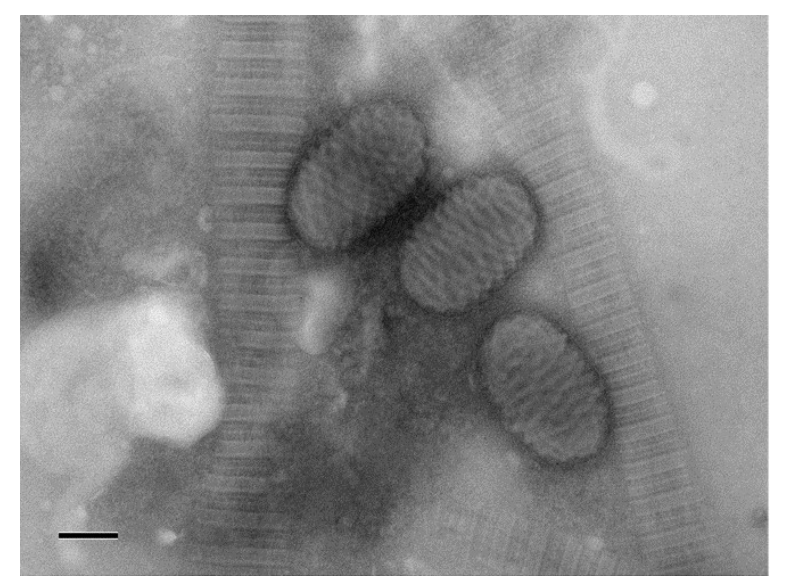

Figure 1. Electron micrograph of Parapoxvirus from caprine oral scab. Negative staining protocol was used. Bar represents $100 \mathrm{~nm}$.

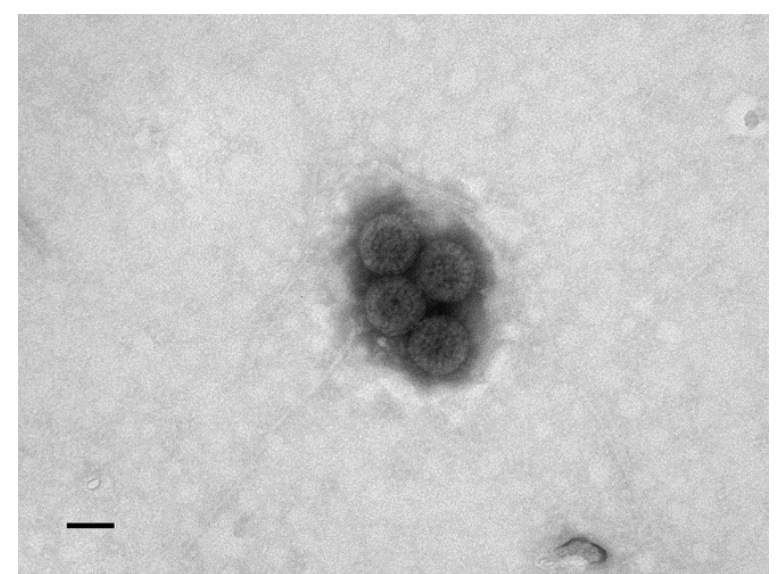

Figure 2. Electron micrograph of Rotavirus from reindeer calf feces. Negative staining protocol was used. Bar represents 50nm. 